\title{
Optimising treatment of schizophrenia: the role of adjunctive fluvoxamine
}

\author{
Siobhan Gee ${ }^{1}$ Oliver Howes ${ }^{2}$
}

Received: 11 November 2015 / Accepted: 12 December 2015 / Published online: 16 January 2016

(C) Springer-Verlag Berlin Heidelberg 2016

Schizophrenia is a leading cause of disability and health burden in adults. In around a third of sufferers, their illness does not respond fully to first or second-line antipsychotic treatment, termed treatment-resistant schizophrenia. For these people, the cost is not only that of continued symptoms but also higher morbidity and indeed mortality. Furthermore, it is also a significant cost to society in lost economic contribution and increased healthcare service use (Kennedy et al. 2014). Clozapine remains the gold standard treatment for treatment-resistant schizophrenia and the only treatment with a really solid evidence base to support its use (Taylor et al. 2015). For a patient who has not responded to two different non-clozapine antipsychotic drugs, the chances of a response with another non-clozapine drug are very low, less than one in twenty, whereas with clozapine, it is one in two (Kane et al. 1988). This is a number needed to treat of 2: an astonishingly low number for any field of medicine and testament to the unique place for clozapine in the treatment of schizophrenia. However, it has a narrow therapeutic window and a number of dose-related side-effects, requiring a complex dose titration (Howes et al. 2012; Beck et al. 2014). It is generally accepted that plasma levels of $>0.35 \mathrm{mg} / \mathrm{L}$ are therapeutic and the risk of toxicity increases markedly with levels above $1 \mathrm{mg} / \mathrm{L}$ (Taylor et al. 2015). Furthermore, levels much above $0.6 \mathrm{mg} / \mathrm{L}$ are associated with

Siobhan Gee

Siobhan.Gee@slam.nhs.uk

1 South London and Maudsley NHS Foundation Trust, Pharmacy Department, Bethlem Royal Hospital, Monks Orchard Road, Beckenham BR3 3BX, UK

2 Institute of Psychiatry, King's College London, Camberwell, SE5 8AF, London, UK an increasing risk of seizures (Taylor et al. 2015). Interpatient variability in clozapine metabolism is considerable, and this combined with the potential for other pharmacokinetic interactions (not least smoking) may make achieving therapeutic plasma levels difficult. Clinicians could prescribe doses above the licensed maximum but if metabolism is rapid, this may simply increase the levels of metabolites and attendant side effects, with little appreciable increase in clozapine levels. Furthermore, dose increases, even within the licensed range, may be unacceptable to patients because of the increased tablet burden. In this context, fluvoxamine added to clozapine treatment may have a special role.

Polypharmacy is usually considered a dirty word in psychiatry. Adding more medicines to established treatments, including to clozapine, usually results in disappointing clinical outcomes (Taylor et al. 2012) and almost invariably adds to the side effect load, long-term physical health risks, and of course, the tablet burden. However, in some circumstances, it may be justifiable. Around 30-50\% of patients do not fully respond to clozapine monotherapy, and negative and depressive symptoms often persist. The article in this edition from Polcwiarkek and Nielsen reviews the use of the antidepressant fluvoxamine as an augmenting agent to clozapine (Polcwiartek and Nielsen 2015). The benefits may be twofold - firstly for residual negative and depressive symptoms. Secondly, fluvoxamine is a potent CYP1A2 inhibitor, and this lends it some characteristics when combined with clozapine that are unusual. Alteration of the metabolic processing of clozapine by fluvoxamine, principally but not exclusively through CYP1A2 inhibition, may increase clozapine plasma concentrations, and possibly lower concentrations of the active metabolite norclozapine. This latter effect is of particular interest for patients who may struggle to tolerate therapeutic plasma concentrations of clozapine, as the metabolites are known to act on different receptor systems to the parent drug, 
and may be responsible for different tolerability problems. It has been additionally suggested that the ratio of clozapine to norclozapine may be of relevance for efficacy.

Polcwiartek and Nielsen describe the successful exploitation of these effects of fluvoxamine in combination with clozapine, but caution is warranted. The magnitude of the effects on clozapine and norclozapine plasma concentrations appears highly variable and unpredictable. The consequences of a sudden rise in clozapine plasma levels may be profound hypotension, tachycardia, sedation, and even seizures. Moreover, as the authors point out, evidence supporting this strategy is often of poor quality, conflicting, and subject to publication bias.

We are clearly lacking good quality trials in this area. We need studies investigating both the metabolic effects of augmenting clozapine with fluvoxamine and the efficacy and tolerability of such strategies. But, in the absence of a better alternative to clozapine, what is the clinician to do now when faced with patients who continue to suffer symptoms or side effects with clozapine monotherapy? The evidence base expertly reviewed by Polcwiartek and Nielsen suggests that adding fluvoxamine is a reasonable treatment approach. However, the risks should be carefully considered and steps taken to reduce potential harm are obviously essential, as is regular formal assessment of the benefits of such interventions. For some patients, fluvoxamine augmentation may be the key to a better tolerated or more effective clozapine regimen. For others, fluvoxamine augmentation may at best be unhelpful, and at worst harmful. Predicting which outcome is more likely is difficult, and so caution must be the maxim.

\section{References}

Beck K, McCutcheon R, Bloomfield MA et al (2014) The practical management of refractory schizophrenia - the Maudsley Treatment REview and Assessment Team service approach. Acta Psychiatr Scand 130:427-438

Howes OD, Vergunst F, Gee S et al (2012) Adherence to treatment guidelines in clinical practice: study of antipsychotic treatment prior to clozapine initiation. Br J Psychiatry 201:481-485

Kane J, Honigfeld G, Singer J et al (1988) Clozapine for the treatmentresistant schizophrenic: a double-blind comparison with chlorpromazine. Arch Gen Psychiatry 45:789-796

Kennedy JL, Altar CA, Taylor DL et al (2014) The social and economic burden of treatment-resistant schizophrenia: a systematic literature review. Int Clin Psychopharmacol 29:63-76

Polcwiartek C and Nielsen J. (2015) The clinical potentials of adjunctive fluvoxamine to clozapine treatment: a systematic review. Psychopharmacology. In press

Taylor DM, Smith L, Gee SH et al (2012) Augmentation of clozapine with a second antipsychotic - a meta-analysis. Acta Psychiatr Scand 125:15-24

Taylor D, Paton C, Kapur S (2015) The Maudsley prescribing guidelines in psychiatry, 12th edn. Wiley-Blackwell, Hoboken 